\title{
Treatment of Myasthenia Gravis
}

Constantine Farmakidis, MD, Mamatha Pasnoor, MD, Mazen M. Dimachkie, MD, Richard J. Barohn, MD

Department of Neurology, University of Kansas Medical Center, 3901 Rainbow Boulevard, Mail

Stop 2012, Kansas City, KS 66160, USA

\section{Keywords}

Myasthenia gravis; Pyridostigmine; Prednisone; Thymectomy; Immunotherapy; Complement inhibition; Intravenous immunoglobulin; Plasma exchange

\section{INTRODUCTION}

Myasthenia gravis (MG) is the most common acquired disorder of neuromuscular transmission. It occurs due to the production of pathogenic autoantibodies that bind to components of the neuromuscular junction, the most common being the acetylcholinesterase receptor (AChR). The incidence is estimated at 0.3 to 2.8 per 100,000 and the worldwide prevalence at 700,000. ${ }^{1}$ In 1934, cholinesterase inhibition was demonstrated as the first effective treatment for $\mathrm{MG}^{2}{ }^{2}$ Until the last 20 years, most MG treatment was investigated through retrospective clinical studies. More recently, there have been a number of randomized controlled clinical trials (Box 1). The decades that various MG treatments were introduced is shown in Box 2. This development has been associated with dramatic improvements in survival and prognosis in $\mathrm{MG}^{3}$ The primary reasons for reduced mortality rates are the improvement in intensive respiratory care and the introduction of immunosuppressive treatments. Although the mortality rate was previously quite high, resulting in the name MG, the current mortality rate in MG is reported as 0.06 to 0.89 per million person-years. ${ }^{4}$ The various treatments for $\mathrm{MG}$ and the approximate time lag to onset of action are outlined in Table 1.

In this review, we summarize information on most MG treatment modalities and offer recommendations for the management of generalized MG and MG crises.

\section{SYMPTOMATIC TREATMENT}

\section{Anticholinesterase Inhibitors}

Acetylcholinesterase inhibitors were discovered and introduced into medical practice during the 19th century. ${ }^{5}$ In 1934, Walker hypothesized that physostigmine, an agent used as a partial antagonist to curare, may counteract the curare poisoning-like features of MG and described rapid onset and dramatic but temporary improvement in a 56-year-old woman with

\footnotetext{
*Corresponding author. mdimachkie@kumc.edu.
} 
generalized MG. ${ }^{2,6}$ She followed this with a brief and also positive report of prostigmine for generalized MG. ${ }^{7}$ Prostigmine was the acetylcholinesterase inhibitor of the time from the mid-1930s to the mid-1950s, when pyridostigmine was introduced. ${ }^{8-11}$ To our knowledge, branded Prostigmin is no longer available in the United States, but generic neostigmine is.

Pyridostigmine, a synthetic acetylcholinesterase inhibitor, inhibits the hydrolysis of the acetylcholine neurotransmitter in the synaptic cleft. This agent increases the number of interactions between the acetylcholine and the acetylcholine receptor in the neuromuscular junction. Pyridostigmine does not cross the blood-brain barrier, thereby limiting central nervous system toxicity, and may be mildly effective in ocular and generalized MG.

A typical starting dose is $60 \mathrm{mg}$ every 6 hours during daytime hours (see Table 1). Dosage may be titrated up to 60 to $120 \mathrm{mg}$ every 3 hours aiming to minimize symptoms, but at these higher doses side effects are more likely to occur. Clinical effect onset is 15 to 30 minutes and its duration is about 3 to 4 hours. For patients who awaken at night or in the morning with impairing weakness, a 180-mg extended release formulation of pyridostigmine may be taken before sleep. However, owing to uneven absorption and unpredictable effect, the use of this medication has been limited.

Gastrointestinal side effects such as abdominal cramping, loose stools, and flatulence are most common. Increased perspiration and muscle twitches and cramps are other side effects. Acetylcholinesterase inhibitors are relatively contraindicated in myasthenic crisis because they can increase secretions and complicate airway management. At very high doses, acetylcholinesterase inhibitors can precipitate a paradoxic increase in weakness with respiratory insufficiency, a condition recognized as a cholinergic crisis. However, in the current era of effective immunotherapy, these extremely high doses are not used, and the cholinergic crisis has become more of a theoretic concern. Pyridostigmine can be used long term, and its effectiveness generally does not diminish over time. For the management of intrusive muscarinic side effects, options include oral glycopyrrolate $1 \mathrm{mg}$, hyoscyamine $0.125 \mathrm{mg}$, or loperamide $2 \mathrm{mg}$. Either drug can be taken concurrently with pyridostigmine doses, up to 3 times a day.

Data exist to guide the use of acetylcholinesterase inhibitors in different MG patient subgroups. Patients with muscle-specific kinase (MuSK) autoantibody-positive disease have lower response rates than patients with the AChR autoantibody. ${ }^{12,13}$ Juvenile patients with MG may have a particularly robust acetylcholinesterase inhibitor response. ${ }^{14}$ Patients with ocular MG, and particularly those with diplopia, frequently seem to not fully respond to acetylcholinesterase inhibitors, although ptosis seems to be more responsive than ocular paresis. ${ }^{15,16}$ The apparent limited response in patients with diplopia may be because, unless the ocular motility is completely restored, some degree of diplopia will persist.

\section{CORTICOSTEROIDS}

Corticosteroid treatment was the first widely used immunosuppressive therapy introduced in MG. The first reports of a beneficial response in MG involved high-dose prednisone (100 $\mathrm{mg} / \mathrm{d}$ or every other day). ${ }^{17,18}$ Early clinical studies showed prednisone's dramatic impact 
on myasthenic patients, with $80 \%$ or more showing either medical remission or marked improvement. ${ }^{19}$ Although evidence from randomized controlled clinical trials remains limited and side effects pose significant challenges in clinical use, corticosteroids are considered the most effective oral immunosuppressive agent and are widely recommended as a first-line agent for use in patients with MG. ${ }^{20-23}$ Although corticosteroids are known to have a broad inhibitory effect on immune response via the reduction of endothelial adhesion of leukocytes and a decrease in inflammatory cytokine production, the exact mechanism of action in MG remains unknown. Studies of the effect of corticosteroids therapy on acetylcholine receptor antibody titers have shown conflicting results with both decreased and unchanged antibody titers. This finding possibly implies an effect on cell-mediated immunity for corticosteroids in MG.

The clinical response to corticosteroids can start within days, and most patients experience initial benefits within the first 2 weeks. ${ }^{19}$ Patients attain maximal improvement on corticosteroids in the first 6 months, although some may take as long as 2 years or more. ${ }^{19}$ There are 2 prevalent approaches to oral corticosteroids administration: a high-dose, rapid treatment induction regimen, and a low-dose and slow titration regimen (see Table 1). The slow titration regimen is designed to reduce the risk of initial worsening seen in as many as one-half the patients started on corticosteroids, but more commonly in the patient subset with severe MG or marked bulbar manifestations. The high-dose regimen consists of prednisone 1.0 to $1.5 \mathrm{mg} / \mathrm{kg} / \mathrm{d}$ (but usually not $>100 \mathrm{mg} / \mathrm{d}$ ) for 2 to 4 weeks. After this period, a decision is made to immediately switch to every other day or to continue daily high-dose therapy. Switching immediately to alternate day high-dose corticosteroids may be used for patients who are Myasthenia Gravis Foundation of America (MGFA) grade 2 (mild). However, higher grade patients with MG usually require daily corticosteroid dosing for extended periods. Whether the patient is switched to a higher daily dosing at 2 to 4 weeks or left on high-dose daily therapy, the patient is usually kept on that dose (eg, $100 \mathrm{mg}$ every other day or $50 \mathrm{mg} / \mathrm{d}$ ) for another 4 to 8 weeks, at which time improvement should be noted and a slow taper by 5 to $10 \mathrm{mg}$ a month can be initiated.

A low-dose and slow titration regimen is suited for patients with milder disability, including ocular MG or in mild to moderate MG. In the low-dose approach, $10 \mathrm{mg} / \mathrm{d}$ is administered, and the prednisone is increased by $10 \mathrm{mg}$ every 5 to 7 days to a peak dose of 1.0 to 1.5 $\mathrm{mg} / \mathrm{kg} / \mathrm{d}$ (up to $60-100 \mathrm{mg}$ ). ${ }^{24} \mathrm{~A}$ third and more recent approach is based on the mycophenolate mofetil study, ${ }^{25}$ and it places patients on a fixed dose of prednisone $20 \mathrm{mg}$ immediately, monitoring that dose, unless there is no response, and then the dose should be increased. We have been using the $20 \mathrm{mg} / \mathrm{d}$ and stay approach since the mycophenolate mofetil study, and have found that it is often successful, as in the mycophenolate study. We believe that a comparative effectiveness study of different prednisone dosing approaches in MG is warranted.

Daily prednisone use is also the rule for patients in myasthenic crisis and for those with worsening symptoms but who are not yet in crisis. A switch to alternate day prednisone can be made months later, when the patient has begun to improve significantly. A daily longterm steroid regimen may be indicated in patients with diabetes and hypertension to avoid wide swings in serum glucose and blood pressure, respectively. 
In ocular MG, the use of corticosteroids has been the subject of debate, weighing the considerable functional impairment from diplopia and ptosis against the risk of significant systemic toxicity from chronic corticosteroid use. ${ }^{26} \mathrm{~A}$ recent small randomized, doubleblind trial of prednisone $10 \mathrm{mg}$ every other day titrated up to $40 \mathrm{mg} / \mathrm{d}$ over 16 weeks versus placebo in patients with ocular MG showed that $100 \%$ of the placebo group patients $(n=5)$ failed to improve, whereas only $17 \%$ of the prednisone group $(\mathrm{n}=6)$ failed to improve $(P=$. 02) ${ }^{20}$ The strength of this evidence is limited by a small sample size, but this study indicates that prednisone can be an effective treatment for ocular MG and should be considered in patients that fail acetylcholinesterase inhibitors. This small but dramatically positive study is probably the best randomized controlled trial of prednisone in MG.

Several retrospective studies have provided evidence that immunotherapy (including treatment with corticosteroids) may reduce the risk of developing generalized MG in patients with ocular MG. ${ }^{27,28}$ In the largest of these studies, after 2 years of follow-up, 36\% of patients not treated on prednisone progressed to generalized MG versus only $7 \%$ of patients treated with prednisone. ${ }^{27}$ In another retrospective study, pyridostigmine was used without prednisone in 59 of 97 patients with ocular MG with 12 developing generalized MG, whereas none of the 38 prednisone-treated cases developed generalized MG. ${ }^{16}$

The systemic side effects of long-term corticosteroid therapy are numerous and can be highly impactful. They include weight gain, diabetes, hypertension, eye disease (cataract and glaucoma), accelerated bone demineralization, and neuropsychiatric disturbances. Potential complications should be discussed before the initiation of treatment, and prevention and monitoring plans should be established in collaboration with the patient's primary care physician. We recommend placing a tuberculin skin test or obtaining a QuantiFERON-TB Gold test to identify patients previously exposed to tuberculosis before starting corticosteroids therapy. Prophylactic therapy is indicated in those who test positive for prior exposure. Patients should be counseled about a low carbohydrate, low calorie, and low salt diet. If the patient is hospitalized, this can be done by the dietician. However, dieticians are often not available in the outpatient setting and, therefore, it is up to the neurologist to provide some dietary guidance. The advice of "no junk food/no salt when food gets to the table" is a good starting point, and should be reinforced on follow-up visits. A dual energy x-ray absorptiometry scan and an ophthalmologic examination should be obtained at baseline and repeated annually. Calcium (500 $\mathrm{mg} 2$ to 3 times daily) and vitamin D (400 IU/d) supplements should be taken to reduce the risk of pathologic fractures. Patients should also remain up to date on all vaccinations, including the flu and pneumococcal vaccines, but no live or live attenuated vaccines should be used by patients on immunotherapy. ${ }^{29}$

\section{OTHER IMMUNOSUPPRESSANTS}

\section{Azathioprine}

Azathioprine is a purine synthesis cytotoxic antimetabolite that inhibits DNA and RNA synthesis, cellular replication, and lymphocyte function. The use of azathioprine for MG therapy was pioneered in Europe in the 1970s, and azathioprine has become the most widely accepted steroid-sparing immunosuppressant used for MG. ${ }^{22,30}$ In comparison with other steroid-sparing options, azathioprine has more favorable tolerability, although a major 
challenge in its clinical use is the estimated 6- to 18-month latency between treatment initiation and therapeutic onset. ${ }^{31,32}$

A number of earlier retrospective studies have suggested response rates to azathioprine ranging from $70 \%$ to $91 \% .^{30,33}$ There has been 1 randomized, double-blind clinical trial of oral prednisolone plus azathioprine $2.5 \mathrm{mg} / \mathrm{kg} / \mathrm{d}$ versus oral prednisolone and placebo. ${ }^{32}$ Enrollment was slow, took several years to complete it. Patients were observed over 3 years and the corticosteroid dose was adjusted up or down to the lowest dose necessary to maintain pharmacologic remission. Thirty-four patients were enrolled, but the dropout rate was high. At 12 months, there was no significant difference in the prednisolone dose between both groups $(\mathrm{N}=24$; placebo 15 cases and azathioprine 9$)$, but there was a trend for a lower prednisolone dose in the azathioprine group. At 18 months, there was a statistically significant difference in the prednisolone dose between the 2 groups. At 3 years, most patients in the prednisolone plus azathioprine group $(n=8)$ had been successfully tapered off steroids. Weight gain was also less in the prednisolone plus azathioprine group compared with the prednisolone and placebo group, at $2 \mathrm{~kg} / \mathrm{y}$ and $5.8 \mathrm{~kg} / \mathrm{y}$, respectively. Conversely, in the prednisolone and placebo groups, patients were more likely to fail to remit and to relapse even with the flaws noted. This is an important positive study in the MG field and supports the use of azathioprine. However, azathioprine may not improve an MG patient in the first year of treatment and is used for long-term management to get patients on lower corticosteroids doses or off corticosteroids altogether.

Azathioprine has been used in patients with generalized MG on corticosteroids who are still symptomatic; in patients with relative contraindications to corticosteroids treatment such as hypertension, diabetes, and osteoporosis; and in those who experience severe side effects to corticosteroids. Azathioprine has also been used in patients with ocular MG requiring but not tolerating corticosteroid therapy. ${ }^{34}$

The starting dose for azathioprine is $50 \mathrm{mg} / \mathrm{d}$ (see Table 1). Dosing can be increased in 50$\mathrm{mg}$ increments every 2 to 4 weeks to a goal dose of 2 to $3 \mathrm{mg} / \mathrm{kg} / \mathrm{d}$. Blood counts and liver function should be tested at baseline, and then monthly. An important monitoring parameter of bone marrow suppression is the white blood count and leukopenia. ${ }^{35}$ Others include liver function test evaluation (alanine aminotransferase, aspartate aminotransferase). We monitor a complete blood count and a complete metabolic panel. If the white blood cell count decreases to less than $4000 \mathrm{~mm}^{3}$, we decrease the azathioprine dose, and if it decreases to less than 3000 per $\mathrm{mm}^{3}$, we stop the drug. We also monitor the absolute neutrophil count to make sure it is not affected, but expect some lymphopenia in the range of 500 to 1000 per $\mathrm{mm}^{3}$. If the aspartate aminotransferase or alanine aminotransferase levels elevate, we stop the drug. When the liver enzymes return to normal the patient can be rechallenged and occasionally this measure can be effective without enzyme elevations.

In rheumatic diseases and in posttransplant care, azathioprine has been linked to a higher risk of developing a malignancy, although a parallel phenomenon has not been described in patients with MG. ${ }^{36}$ Although evidence from the transplant literature indicates that the risk for adverse outcomes from azathioprine use in pregnancy is very low, we do not use azathioprine in pregnancy. 
Of the patients placed on azathioprine, $10 \%$ to $20 \%$ have an idiosyncratic drug reaction presenting as a flulike syndrome with fever, malaise, and loss of appetite. ${ }^{29}$ This phenomenon occurs in the first 1 to 2 weeks after starting the drug. If it occurs, azathioprine should be stopped immediately, and the symptoms will lessen in a day or two. If azathioprine is restarted, these side effects almost always recur.

It has been suggested that before initiation of azathioprine, thiopurine methyltransferase phenotype or genotype be tested as an inherited enzyme deficiency predicts an increased risk for leukopenia. A systematic review of 55 studies found that, although diminished TMPT activity is associated with myelotoxicity, there is insufficient evidence to support screening patients for thiopurine methyltransferase deficiency. ${ }^{37}$ In practice, we monitor blood cell counts closely instead.

\section{Mycophenolate Mofetil}

Similar to other newer immunosuppressants, mycophenolate mofetil was introduced in neuromuscular diseases after initial experience as an antirejection drug in transplant medicine. ${ }^{38}$ Mycophenolate mofetil is a potent monophosphate dehydrogenase inhibitor. It inhibits guanosine nucleotide synthesis that is essential for B and T lymphocytes. Initial interest was spurred in MG after the report of a patient with treatment-refractory early-onset myasthenia who had a rapid response to mycophenolate mofetil. ${ }^{39}$ Several retrospective studies suggested a favorable tolerability profile, the potential for a prednisone-sparing effect, and robust rates of disease control around $70 \% .{ }^{40,41}$ In addition, in comparison with azathioprine, a more rapid initial clinical response time (11 weeks) was suggested.

However, both of 2 large multicenter, randomized, double-blinded, placebo-controlled trials failed to show that mycophenolate mofetil in addition to prednisone was more effective in controlling MG. In 1 study, 80 patients with mild to moderate generalized AChR antibodypositive MG were randomized to $20 \mathrm{mg} / \mathrm{d}$ of prednisone plus $2.5 \mathrm{~g} / \mathrm{d}$ mycophenolate mofetil versus $20 \mathrm{mg} / \mathrm{d}$ prednisone and placebo and followed over 12 weeks. ${ }^{25}$ The primary outcome was change in the Quantitative Myasthenia Gravis (QMG) score, which was similarly decreased in both groups, indicating there was no advantage detected in the mycophenolate mofetil group. Both groups improved which implies a significant effect of prednisone $20 \mathrm{mg} / \mathrm{d}$. In the international phase III mycophenolate mofetil study, $176 \mathrm{AChR}$ antibody-positive patients with mild to moderate MG who were already taking corticosteroids were randomized to mycophenolate mofetil $2 \mathrm{~g} / \mathrm{d}$ versus placebo. ${ }^{42}$ At the conclusion of 36 weeks ( 9 months), the primary endpoint measured - which was a composite of a favorable MGFA postintervention status and prednisone and pyridostigmine doses below certain preset ceiling levels — did not show the mycophenolate mofetil group outperforming the placebo group.

The discordance between the retrospective and randomized trial data of mycophenolate mofetil has several potential explanations. The most favored is that the therapeutic potency of $20 \mathrm{mg}$ of prednisone may have been underestimated and thus overwhelmed the therapeutic effect of mycophenolate mofetil. It is also possible that clinical trial periods were not long enough to capture the onset of the effect of mycophenolate mofetil, or that the disease population studied was too mildly affected to require both prednisone and 
mycophenolate mofetil for treatment. Since the publication of these negative randomized, controlled trials, another retrospective study provided evidence of benefit for mycophenolate mofetil, although the strength of the evidence is limited by its retrospective design. ${ }^{43}$ Despite 2 negative studies, mycophenolate mofetil is listed as part of the international consensus guidance for MG management. ${ }^{22}$ In our practice, although we still use mycophenolate mofetil for some patients with MG, we do not use it quite as often since the publication of these 2 randomized controlled trials. The most common regimens used are 1000 to $1500 \mathrm{mg}$ twice daily (see Table 1). The main side effects are diarrhea, nausea, infections, and leukopenia. Blood counts should be monitored closely at the initiation of treatment and thereafter monthly, and we use the same guidelines for dosing adjustment outlined for azathioprine. Mycophenolate mofetil is contraindicated in pregnancy owing to teratogenic potential and a higher risk of miscarriage in the first 3 months. ${ }^{44}$ Concerns exist regarding a potential increase in the risk of lymphoproliferative disease based on isolated case reports. ${ }^{45,46}$

\section{Cyclosporine}

Cyclosporine, an agent first used to suppress allograft rejection, interferes with calcineurin signaling, suppresses cytokine secretion including interleukin- 2 and interferon- $\gamma$, and interferes with T-helper cell activation. Cyclosporine was the first immunosuppressant medication shown to be effective in the treatment of generalized MG in 2 small doubleblind, randomized, controlled trials. ${ }^{47,48}$

In the first randomized trial, newly diagnosed, thymectomy- and immunosuppression-naïve generalized patients with MG were treated with cyclosporine $6 \mathrm{mg} / \mathrm{kg} / \mathrm{d}$ versus placebo. The cyclosporine level was monitored, and the dose adjusted to maintain trough levels between 400 and $600 \mathrm{ng} / \mathrm{mL}$ and creatinine at $2.0 \mathrm{mg} / \mathrm{dL}$ or less. At 6 months, the cyclosporine group had a lower QMG score compared with the placebo group, and that persisted and remained statistically significant at 12 months. ${ }^{47}$ In a second randomized, controlled trial of cyclosporine, a group of steroid-dependent patients ( $230 \mathrm{mg}$ of prednisone every other day) with or without a thymectomy, and with varying degrees of prior immunosuppressive therapy was treated with $5 \mathrm{mg} / \mathrm{kg} / \mathrm{d}$ of cyclosporine versus placebo with the cyclosporine dose adjusted to maintain trough levels between 300 and $500 \mathrm{ng} / \mathrm{mL}$ and creatinine of 2.0 $\mathrm{mg} / \mathrm{dL}$ or less. ${ }^{48}$ At the conclusion of the study at 6 months, the cyclosporine group had a lower QMG score, had a greater reduction of AChR antibody levels, and was on a lower prednisone dose, although this lower dose was not statistically significant. In an 18-month, open-label extension of the study, the steroid-sparing effect of cyclosporine seemed to increase.

Acute and more indolently progressive renal toxicity and hypertension are major factors limiting the tolerability of cyclosporine. Serum creatinine levels in a case series increased by a mean of $48 \%$ in more than one-quarter of treated patients and the cumulative side effects led to the discontinuation of treatment in $35 \%$ of patients over a 2 -year period. ${ }^{48,49}$ There is also evidence that cyclosporine is associated with increased dermatologic and other malignancy risk. ${ }^{49}$ In addition to increased skin surveillance and measures to limit sun exposure, the neoplasia risk of cyclosporine should be reviewed individually before 
initiating treatment. Other limiting side effects are hirsutism, tremor, gum hyperplasia, paresthesias, headaches, and hepatotoxicity.

The starting dose of cyclosporine is usually $3 \mathrm{mg} / \mathrm{kg} / \mathrm{d}$ (see Table 1) and it comes in $100 \mathrm{mg}$ capsules. Thus, a 70-kg person generally takes $200 \mathrm{mg}$ split in 2 doses. Similar to corticosteroids, the goal is to reduce cyclosporine to the lowest dose that maintains treatment effect. Trough levels should be monitored (keep at $<300 \mathrm{ng} / \mathrm{mL}$ ) as well as serum creatinine, blood urea nitrogen, and liver function tests. Different cyclosporine preparations should not be mixed owing to differing pharmacokinetics, and the patient' medication lists should be screened before the initiation of this drug because a number of medications interact with cyclosporine and destabilize serum drug levels.

Tacrolimus, a similar agent to cyclosporine, also seems to have a beneficial effect in MG, as shown in a small randomized pilot study. ${ }^{50}$ In another study, a cohort of 13 children aged 7 to 13 years were treated for 1 year with tacrolimus 1 to $2 \mathrm{mg} / \mathrm{d}$ for MG poorly responsive to prednisone. ${ }^{51}$ The prednisone dose was significantly decreased, with improvement in MG symptoms as assessed by the QMG, MG Manual Muscle Testing, and MG Activities of Daily Living and reduction of anti-AChR antibody titers. Most patients were able to completely discontinue prednisone.

\section{Methotrexate}

Methotrexate is a folate antimetabolite that inhibits dihydrofolate reductase. When given in high doses as part of a cancer chemotherapy regimen, methotrexate has a distinct cytotoxic effect; at lower doses, methotrexate induces an immunomodulatory effect, the mechanism of which is not fully understood. ${ }^{52} \mathrm{~A}$ small randomized, single-blinded study of methotrexate in MG compared methotrexate $17.5 \mathrm{mg} / \mathrm{wk}$ with daily prednisone as compared with azathioprine at 2.5 to $3.0 \mathrm{mg} / \mathrm{kg} / \mathrm{d}$ with daily prednisone. ${ }^{53}$ At 2 years there was a substantial and comparable decrease in the average daily prednisone dose and the QMG scores in both groups. These data suggested a similar efficacy between azathioprine and methotrexate over a 2-year period, although with a cost advantage for methotrexate. A randomized, doubleblind, placebo-controlled trial of methotrexate $20 \mathrm{mg} / \mathrm{wk}$ by mouth versus placebo in prednisone-dependent patients with MG was designed to more definitively determine if methotrexate is effective as a corticosteroid-sparing agent. ${ }^{54}$ The results using the predetermined intention-to-treat multiple imputation analysis showed no difference in the prednisone area under the curve between methotrexate and placebo over a 12-month observation period. Primary analysis of the secondary outcomes (QMG, MG Activities of Daily Living, etc) similarly showed no difference between the 2 groups. However, there were more patients in the placebo group that dropped out owing to worsening MG. In addition, a post hoc analysis using other intention-to-treat methods (last-dose-carried forward, worst/highest dose carried forward) showed methotrexate patients had significantly lower QMG, MG Activities of Daily Living and MG Composite scores (Table 2).

As in the mycophenolate trials, this study raised the question of whether the drug is ineffective, or whether the trial's sensitivity was limited by concurrent corticosteroids treatment, insufficiently long follow-up, a small study sample, or incorrectly chosen intention-to-treat design. 
As a third-line agent, methotrexate is started at $10 \mathrm{mg} / \mathrm{wk}$ and titrated to $20 \mathrm{mg} / \mathrm{wk}$ over 2 months (see Table 1). We also give folic acid $1 \mathrm{mg} / \mathrm{d}$ to prevent stomatitis and monitor for bone marrow suppression and liver toxicity. Methotrexate is strictly contraindicated in women who may become pregnant and should be used cautiously in patients with lung pathology because it is rarely associated with pulmonary fibrosis.

\section{Cyclophosphamide}

Cyclophosphamide is an alkylating agent that modifies the guanine base of DNA, conferring cytotoxic properties. This action in turn suppresses bone marrow cell replication and B- and T-cell immune function. A case series and a small, randomized double-blind clinical trial have provided evidence that cyclophosphamide both improves weakness and also has steroid-sparing effect in $\mathrm{MG}^{55,56}$

In a randomized, controlled trial of $500 \mathrm{mg} / \mathrm{m}^{2}$ monthly intravenous cyclophosphamide pulses, those in the cyclophosphamide arm had a significantly improved QMG score at month 12 and a lower steroid dose at months 6 and $12 .{ }^{56}$ Drachman and associates ${ }^{57}$ described long-lasting improvement in 3 patients with refractory disease treated with "rebooting of the immune system" through intravenous cyclophosphamide $50 \mathrm{mg} / \mathrm{kg}$ for 4 days, followed by rescue with granulocyte colony stimulating factor. The associated toxicity is, however, considerable with alopecia reported in $75 \%$, leukopenia in $35 \%$, and nausea and vomiting in $25 \%$ of patients and the increased risk of hemorrhagic cystitis. ${ }^{55}$ Cyclophosphamide remains an option for severe and refractory MG. However, owing to a poor tolerability profile and the advent of alternative immunotherapy, cyclophosphamide is used only rarely for MG.

\section{Rituximab}

Rituximab is a genetically engineered chimeric mouse-human monoclonal antibody directed against CD20, a transmembrane protein selectively found on the surface of normal and malignant B-lymphocytes. ${ }^{58}$ Rituximab decreases the number of circulating CD20 $\mathrm{B}$ cells and is also thought to suppress antibody production and humoral immunity. A case of a treatment-resistant MG patient with an apparent response to rituximab provided initial evidence that rituximab may have a role in $\mathrm{MG}$ treatment. ${ }^{59}$

Rituximab therapy in MG is supported by demonstrable defects in B-cell tolerance checkpoints in MG. ${ }^{60}$ These investigators identified defects in B cells, some of which were large-scale abnormalities in B-cell antibody repertoires that were unique to either AChR MG or MuSK MG. These findings suggest that the repertoires reflect the distinct properties of these 2 MG subtypes and that perhaps treatment response may be different in AChR MG from MuSK MG. Nonetheless, retrospective reports have provided additional evidence for a role for rituximab in MG. ${ }^{61}$ In patients with MuSK MG, a particular subgroup otherwise known to be less responsive to standard therapies, retrospective data suggest that rituximab may have a more robust and persistent treatment effect. ${ }^{62,63}$

A recent systematic review of available retrospective rituximab studies found that the Modified MFGA postintervention scale of minimal manifestation status or better was attained in $72 \%$ of MuSK patients, $30 \%$ of AChR antibody patients, and $44 \%$ in both groups 
combined. ${ }^{58}$ The strongest predictors for a clinical response were a positive MuSK antibody status, less severe disease, and younger age at the time of treatment.

The optimal rituximab dosing for MG is not established. A commonly used induction regimen is $375 \mathrm{mg} / \mathrm{m}^{2}$ infusions given weekly for 4 weeks (see Table 1). ${ }^{58,64}$ Another method that we often use is to administer $1 \mathrm{~g}$ and in 2 weeks administer another 1-g dose. Patients can be redosed every 4 to 6 months, but for how long is not known. Also unknown is the benefit of measuring B-cell counts (CD20) before the next dose is given. We do not do this routinely. Progressive multifocal encephalopathy (PML) is a feared complication of rituximab therapy that occurs after reactivation of the JC virus. To date, only 1 patient has been reported with PML in the setting of rituximab therapy for MG, and notably in the setting of prior longstanding use of other immunosuppresants. ${ }^{65} \mathrm{~A}$ recent study reported a large series of PML cases in the setting of rituximab and natalizumab therapy, mostly for lymphoproliferative and rheumatic diseases. ${ }^{66}$ This study suggested that older age and male sex are risk factors for developing PML.

A multicenter randomized, controlled trial of rituximab in generalized MG has completed recruitment. ${ }^{67}$ The primary outcome measure investigated is the percent of patients achieving a $75 \%$ or greater reduction in the mean daily steroid dose recorded over the last month of a 12-month follow-up period and frequency of study-related adverse events.

\section{RAPID-ACTING IMMUNOTHERAPIES}

\section{Plasma Exchange}

Plasma exchange (PLEX) has garnered wide acceptance as an effective treatment in patients with MG since initial reports of its use in the late $1970 \mathrm{~s} .{ }^{68,69}$ Unfortunately, no adequate randomized, controlled trial has been performed to evaluate whether PLEX improves longor short-term outcomes in MG; however, there is indirect evidence for benefit. While early in the use of plasmapheresis for neuromuscular disease, a randomized Guillain-Barré Syndrome study was done in North America comparing plasmapheresis with care without plasmapheresis. ${ }^{70}$ Such a study was never done in MG. This was highlighted in the American Academy of Neurology Therapeutic and Technology Awareness Subcommittee, which gave PLEX in MG crisis a level U (unknown whether it is effective or not) recommendation based on class III evidence. ${ }^{71}$ Several randomized studies comparing the efficacy of PLEX with intravenous immunoglobulin (IVIG) showed that IVIG and PLEX had comparable therapeutic in patients with moderate to severe disease, and a few years earlier IVIG had been shown to be independently superior to placebo in MG. ${ }^{72-74}$ Indications for a short-term course of PLEX are crises (MG grade 5, on mechanical ventilation), impending crisis in patients with severe MG (grade 4/4B) with dysphagia, respiratory dysfunction, or generalized weakness and when a patient with mild (2/2B) or moderate (3/3B) MG is worsening or not responding to other immunosuppressant therapies. An additional indication is prethymectomy in symptomatic patients to treat respiratory and bulbar weakness before surgery. In patients with highly refractory MG, chronic PLEX can be useful in long-term disease control, although no standard chronic treatment protocols have been evaluated systematically. 
Venous access can be peripheral or central, although when adequate peripheral venous access is available it is preferable owing to the lower risks of peripheral vein cannulation. One standardized regimen used in clinical studies consists of 5 PLEX procedures where 1 plasma volume is exchanged per procedure and treatments occur every other day (see Table 1). ${ }^{75}$ The replacement fluid used for plasma is $5 \%$ albumin with added calcium gluconate to prevent hypocalcemia and its clinical sequelae, known as the citrate effect. For patients who require central venous access, PLEX treatments may also be performed daily over 5 days to reduce the risk of a catheter-related infection. ${ }^{75}$

PLEX's mechanism of action is through the removal of plasma-soluble factors, including pathogenic autoantibodies and cytokines. ${ }^{76}$ Clinical improvement typically starts by the third treatment. The rapid onset of treatment effect suggests PLEX may be a preferred intervention when a patient is rapidly worsening. The treatment effect lasts in the order of weeks and provides a window for intensifying immunosuppressive therapy. In a controlled trial of PLEX in patients with MG, at day 14 after a full course of PLEX, 65\% of patients improved. ${ }^{73}$

Recently, additional considerations in the use of PLEX have emerged. A cross-sectional analysis of patients with MG in a nationwide inpatient database from the United States treated with PLEX suggested that a greater than 2-day delay after admission in PLEX administration was associated with higher mortality and complication rates. ${ }^{77}$ Furthermore a single-center, retrospective analysis of a 33-year experience with PLEX and IVIG in juvenile MG, suggested that unlike in adult-onset MG where IVIG and PLEX are thought to be comparable, in juvenile MG, response to PLEX is more consistent. ${ }^{78}$

Traditionally, PLEX has been viewed as difficult to prescribe, complicated to deliver, and limited by central catheter-related complications such as infection, pneumothorax, and thromboembolism, in addition to milder side effects such as fever, urticaria, hypocalcemia, and hypotension. Prospective data from 1727 successive PLEX treatments in 174 patients (13\% with MG), however, showed that complications, although not infrequent, are minor and with very few treatment discontinuations or transitions to a higher level of care. ${ }^{79}$ Similarly, a subanalysis of the PLEX arm in a single-center prospective PLEX and IVIG comparison study indicated that PLEX has the potential for very good tolerability when delivered in a center with significant expertise. ${ }^{75}$ Specifically, $90 \%$ of patients with moderate to severe MG received PLEX as outpatients, $83 \%$ of patients completed PLEX via peripheral venous access, and adverse reactions were generally mild. In patients who require long-term PLEX and have difficult peripheral access, we have inserted arteriovenous fistulas in the arms with some success (Fig. 1). ${ }^{80}$

\section{INTRAVENOUS IMMUNOGLOBULINS}

Early uncontrolled studies suggested that IVIG is a safe and effective adjunctive treatment for MG. ${ }^{81-83} \mathrm{~A}$ first randomized trial of IVIG in MG was cut short owing to logistical reasons (nationwide shortage of IVIG) and was inconclusive. ${ }^{84}$ After demonstrating that PLEX and IVIG are equivalent therapies in MG acute exacerbation, ${ }^{85}$ Gajdos and colleagues ${ }^{86}$ reported in 2005 no superiority of IVIG $2 \mathrm{~g} / \mathrm{kg}$ over $1 \mathrm{~g} / \mathrm{kg}$ in treating acute 
MG exacerbation. A second randomized, double-blind, placebo-controlled trial compared the effect of $2 \mathrm{~g} / \mathrm{kg}$ of IVIG over 2 days with an equivalent volume of placebo infusion in patients with MG with worsening weakness. The study, which was reported in 2007, found meaningful clinical improvement at 14 days via the QMG score in the IVIG group, although the magnitude of the improvement was surprisingly small. The potential for IVIG benefit effect may have been underreported, however, because many patients with milder disease were included in the study cohort. A subgroup analysis underscored this possibility, showing that only patients with moderate to severe disease had a significant treatment effect. ${ }^{74}$ Nevertheless, to date this is the only positive randomized, controlled trial comparing IVIG with placebo for MG. Currently, trials are underway by the pharmaceutical industry that, if positive, could lead to labeling indication from the US Food and Drug Administration of IVIG for MG.

IVIG has a complex immunomodulatory mechanism of action and almost every component of the immune system is involved: IVIG interferes with costimulatory molecules, suppresses antibody production, hinders complement activation and MAC formation, and modulates the expression of $\mathrm{Fc}$ receptors on macrophages and diminishes chemokine, cytokine and adhesion molecule synthesis. ${ }^{87}$

The indications for the use of IVIG in MG are identical as with PLEX. The induction dose is $2 \mathrm{~g} / \mathrm{kg}$ divided over 2 to 5 days (see Table 1), but typically we do the induction over 2 to 3 days, unless the patient is hospitalized. A variety of complications have been reported with the use of IVIG in neuromuscular diseases, but most are mild to moderate in severity. ${ }^{88}$ Prospective studies of IVIG use in neuromuscular disease have shown that headache is common, but that the incidence of serious adverse events is minimal. ${ }^{74}$ Acute renal failure is uncommon and related to patient dehydration and the prior use of sucrose or maltose diluents. Other severe and rare reactions are anaphylaxis, stroke, myocardial infarction, deep venous thrombosis, and pulmonary emboli.

\section{ECULIZUMAB}

Complement has been known to have a crucial role in the pathogenesis of $\mathrm{MG},{ }^{89-91}$ leading to the hypothesis that inhibiting various stages of the complement cascade could lead to clinical improvement in MG. Eculizumab is a recombinant humanized monoclonal antibody that binds to the $\mathrm{C} 5$ complement protein and inhibits its subsequent cleavage and formation of the C5b-9 membrane attack complex. It was recently approved in late 2017 for the treatment of adult patients with generalized MG who are AChR antibody-positive after successful trials. ${ }^{92}$ Candidates for this novel therapy are those in a moderate/severe status category despite receiving adequate trials with most if not all of the discussed immunotherapies. The drug is given via intravenous infusion with a recommended dosage regimen of $900 \mathrm{mg} / \mathrm{wk}$ for the first 4 weeks, $1200 \mathrm{mg}$ for the fifth week, and $1200 \mathrm{mg}$ every 2 weeks thereafter (see Table 1). Eculizumab requires meningococcal vaccination before starting therapy. The introduction of complement inhibition could dramatically change how we manage patients with MG. Other drugs that inhibit complements are currently under study for MG. 


\section{SURGICAL TREATMENT: THYMECTOMY}

Thymectomy has a central role in the treatment of MG. In thymomatous MG, the tumor should be removed. Tumor histologic grade, excision margins, and any distal spread guide treatment decisions regarding any subsequent radiation, chemotherapy, and monitoring. Along with thymoma, the entirety of the thymus tissue should be removed. Improvement in myasthenic symptoms may or may not follow. In multimorbid patients with high operative risk, palliative radiation therapy as an alternate can also be considered. ${ }^{22}$

In nonthymomatous generalized MG, thymectomy has become the standard despite a lack of evidence from a good prospective clinical trial. Two systematic reviews of the existing thymectomy literature emphasized this knowledge gap and recommended the MG field perform a randomized, controlled trial. ${ }^{93-95}$ However, owing to the difficulty of performing controlled trials involving thoracic surgery in a rare disease, high-quality evidence about thymectomy had been lacking. A recently completed landmark international, randomized, rater-blinded clinical trial controlling for medical treatment was designed to address this uncertainty. ${ }^{96}$ One hundred twenty-six recently diagnosed patients, ages 18 through 65 with AChR antibody-positive generalized MG were randomized to receive either extended transsternal thymectomy plus prednisone versus medical management with prednisone. Over a 3-year follow-up period, the time-weighted average QMG score was lower in the patients who underwent thymectomy $(6.15$ vs $8.99 ; P<.001)$. Similarly, the thymectomy group had a lower time-weighted alternate-day prednisone dose requirement (initially reported at $44 \mathrm{mg}$ vs $60 \mathrm{mg}$; $P<.001)$, which was later corrected to $32 \mathrm{mg}$ versus $54 \mathrm{mg}$ (95\% confidence interval, $12-32 \mathrm{mg} ; P<.001)$ Fig. 2 . Also in the thymectomy group, there were fewer patients requiring additional immunosuppression, fewer adverse events, and fewer admissions for myasthenic crises.

These data provide support for thymectomy as a first-line treatment modality that can improve MG status and decrease the required dose and duration of immunotherapy in generalized MG. The operation should be scheduled when the patient is neurologically optimized, because perioperative events can exacerbate myasthenic weakness. Patients with persistent bulbar, respiratory, or limb weakness should be treated with PLEX before surgery. Surprisingly, the effects of the thymectomy could be observed as early as 3 to 4 months and were maintained for the entire 3-year study.

Thymectomy in MuSK, LRP4, and agrin antibody-positive patients is not supported by current evidence. ${ }^{22}$ Patients with MG with MuSK antibodies were not included in the recent thymectomy study. Nevertheless, MuSK and "double-negative" antibody patients have undergone thymectomy and have done well. ${ }^{14}$ Similarly, there is limited evidence to support thymectomy in patients with ocular MG, although if the patient is AChR antibody positive, it may be considered in refractory cases. ${ }^{97}$

The recently completed thymectomy trial mandated a sternal-splitting procedure. Several new less invasive procedures are now being used for thymus removal (Table 3). Videoassisted thoracoscopic surgery and robotic approaches to thymectomy such as robotic videoassisted thoracoscopic surgery offer shorter hospital durations of stay and limited morbidity 
have emerged as alternatives to the classic transsternal approach. ${ }^{98,99}$ There are no trials comparing these surgical techniques, however, and available reports suggest comparable results.

\section{TREATMENT STRATEGIES FOR GENERALIZED MYASTHENIA GRAVIS}

The vast majority of patients with MG improve with therapy over time. Some can often go into remission or minimal manifestation status. ${ }^{100}$ For refractory patients, obtaining care in specialized centers is likely particularly beneficial. A complete remission is defined as having no symptoms or signs and being off all medications for 2 years. Pharmacologic remission is also no symptoms or signs for 2 years, but on stable medication doses. Minimal manifestation status indicates no symptoms, but includes minimal clinical signs such as mild orbicularis oculi or hip flexor weakness (which may never fully resolve).

Fig. 3A summarizes our suggested treatment algorithm for generalized MG. First-line treatment is acetylcholinesterase inhibitors. Simultaneously, the patient should be considered for thymectomy. If a patient remains symptomatic on pyridostigmine, then it is probably time to initiate corticosteroid therapy. We consider acetylcholinesterase inhibitors, corticosteroids, and thymectomy all first-line therapies for generalized MG.

All newly diagnosed patients with MG should have a chest computed tomography scan to assess for thymoma. For patients with thymoma, thymectomy should be done immediately or as soon as the patient is strong enough after initiating immunomodulatory treatment to undergo surgery. We want to emphasize that the chest computed tomography scan is done to search for thymoma and not for thymic "hyperplasia" to decide if a thymectomy should be done in nonthymomatous patients. The decision for thymectomy in nonthymomatous patient is not based on the results of the chest computed tomography scan.

If the patient does not progress to a minimal manifestation status or remission, additional immune therapy should be considered until disease control is attained. Typically, patients with generalized disease require pyridostigmine with prednisone for the initial control of their disease, because pyridostigmine is not enough. For patients with severe weakness at presentation, or if they are diabetic, a steroid-sparing agent such as azathioprine may be started simultaneously with prednisone. If the patient worsens after a prednisone taper, second-line immunosuppressive therapy with azathioprine can be added at that time, realizing that the full benefit of azathioprine therapy may not occur for 12 to 18 months. If an agent that works faster is preferred, then IVIG or cyclosporine (or tacrolimus) are the other second-line choices that have been shown to be effective in randomized, controlled trials (Table 4). We use IVIG as a second-line immunosuppressive agent and usually in a patient who has improved but still has symptoms and signs of MG. We do not use IVIG as a first-line treatment, although the results of ongoing trials of IVIG could alter our practice. Third- and fourth-line options are plasmapheresis, mycophenolate mofetil, methotrexate, and rituximab, and can be used subsequently. In patients who have not responded to these therapies, we discuss chronic therapy with eculizumab infusions every other week. 
At this time, we are considering eculizumab use in patients who are on prednisone and have tried 1 or more additional immunosuppressive drugs with incomplete disease control. In part, this decision is based on not having enough experience with the drug in our clinics and in part owing to the significant expense of the drug. Interestingly and surprisingly the US Food and Drug Administration approved labeling indication is for generalized MG with no requirement that the patient is on any other immunosuppressant therapy.

There are limited trial data to guide tapering of immune therapies in patients who have attained minimal manifestation status or pharmacologic remission. High-dose corticosteroid therapy started early in the course of MG should be considered for tapering 1 to 2 months after the patient has begun to improve. The goal is to try to get patients off prednisone if possible after 1 year or so of therapy. Sometimes, this maneuver is possible, but sometimes patients need to be left on a small dose of prednisone to prevent a relapse such as 5 to 7.5 $\mathrm{mg} / \mathrm{d}$ or every other day. This determination can only be made by trial and error. After the patient has tapered off prednisone, then the steroid-sparing agents can also be tapered. Generally, we try to taper off prednisone first, leaving the patient on the second agent for a period of time (a year or two) before we attempt to slowly taper off the steroid-sparing agent.

\section{TREATMENT STRATEGIES FOR MYASTHENIC CRISIS}

MGFA grade 5 is a myasthenic crisis in which a patient is on mechanical ventilation. For patients in impending crises requiring intubation, abnormal blood gas levels cannot be relied on because they are insufficiently sensitive to impeding respiratory failure. Owing to the nature of myasthenic fatigability, clinical decline can be rapid and unexpected. Patients should be closely monitored for paradoxic breathing, orthopnea, diaphoresis, and a decline in pulmonary function via vital capacity and negative inspiratory force testing.

The treatment of MG crisis consists of rapid immunotherapy with either IVIG or PLEX. Concurrently, patients should be evaluated for infection and other precipitating events, such as the use of medications that can exacerbate MG. Because the effects of IVIG or PLEX are limited to several weeks, long-term immunosuppression should be intensified simultaneously and most frequently with prednisone, up to $100 \mathrm{mg} / \mathrm{d}$ or the methylprednisolone intravenous equivalent. Although acetylcholinesterase inhibitors are available intravenously, they should not be given in the setting of a crisis because they can increase respiratory secretions and complicate airway management. Therefore, all acetylcholinesterase inhibitors are stopped while the patient is intubated. This step is not because of the possibility of cholinergic crisis, which, as we stated, does not occur in the modern era with routinely used does of acetylcholinesterase inhibitors.

We do not have optimal data on the use of IVIG versus PLEX in myasthenic crisis. Gajdos and colleagues in France ${ }^{85,86}$ and then Bril and colleagues in Canada ${ }^{62}$ performed comparative effectiveness studies of IVIG and PLEX in moderate and severe MG and found the treatments to be equivalent. However, a few of these patients were in actual crises on a ventilator. A small controlled cross-over study of IVIG and PLEX showed similar efficacy in MG but faster onset of improvement at 1 week with PLEX. ${ }^{101}$ In addition, a retrospective 
study in juvenile MG showed a more consistent response to PLEX that IVIG. ${ }^{78}$ The selection between these treatments often depends on availability and institutional experience in addition to individual patient factors. Fig. 3B summarizes our suggested treatment algorithm for myasthenic crisis. The pros and cons of IVIG versus PLEX are shown in Table 5 .

Once a patient is on a ventilator, typically they need to be mechanically ventilated for 5 to 7 days. Extubating a patient after only a few days of mechanical ventilation often results in reintubation. Therefore, a conservative approach to extubation is recommended in this setting.

\section{Emerging Therapies}

There are other drugs that inhibit complement currently under study for MG. A phase II industry trial of belumimab, a monoclonal antibody against B-cell activating factor, was just completed with results pending. A multicenter investigator initiated subcutaneous gamma globulin study in MG () is underway with the University of Kansas as the primary organizing site. A phase II study with a drug that increases muscle contractions, tirasemtiv, to improve strength in patients with MG was recently completed with some encouraging results. ${ }^{102}$ As noted, the results of the National Institutes of Health-funded rituximab study in generalized MG will be released in 2018 ().

\section{Acknowledgments}

Disclosure Statement: Drs C. Farmakidis and M. Pasnoor have nothing to disclose. Dr M.M. Dimachkie is on the speaker's bureau or is a consultant for Alnylam, Baxalta, Catalyst, CSL-Behring, Mallinckrodt, Novartis, NuFactor, and Terumo. He has also received grants from Alexion, Biomarin, Catalyst, CSL Behring, FDA/OPD, GSK, Grifols, MDA, NIH, Novartis, Orphazyme, Sanofi, and TMA. Dr R.J. Barohn is a consultant for NuFactor and is on the advisory board for Novartis. He has received an honorarium from Option Care and PlatformQ Health Education. He has received research grants from NIH, FDA/OOPD, NINDS, Novartis, Sanofi/Genzyme, Biomarin, IONIS, Teva, Cytokinetics, Eli Lilly, PCORI, ALSA, and PTC. This work was supported by a CTSA grant from NCATS awarded to the University of Kansas for Frontiers: University of Kansas Clinical and Translational Science Institute (\# UL1TR002366) The contents are solely the responsibility of the authors and do not necessarily represent the official views of the NIH or NCATS.

\section{REFERENCES}

1. Deenen JC, Horlings CG, Verschuuren JJ, et al. The epidemiology of neuromuscular disorders: a comprehensive overview of the literature. J Neuromuscul Dis 2015;2(1):73-85. [PubMed: 28198707]

2. Walker MB. Treatment of myasthenia gravis with physostigmine. Lancet 1934; 223(5779):1200-1.

3. Grob D, Brunner N, Namba T, et al. Lifetime course of myasthenia gravis. Muscle Nerve 2008;37(2):141-9. [PubMed: 18059039]

4. Carr AS, Cardwell CR, McCarron PO, et al. A systematic review of population based epidemiological studies in myasthenia gravis. BMC Neurol 2010;10:46. [PubMed: 20565885]

5. Proudfoot A The early toxicology of physostigmine: a tale of beans, great men and egos. Toxicol Rev 2006;25(2):99-138. [PubMed: 16958557]

6. Walker MB. The James Lind Library: treatment of myasthenia with Physostigmine. Video of original Mary Walker patient treated with physostigmine. 1934 Available at: http:// www.jameslindlibrary.org/walker-mb-1934/. Accessed June 13, 2017.

7. Walker M Case showing the effect of prostigmin on myasthenia gravis. Proc R Soc Med 1935;28:759-61. 
8. Osserman KE, Teng P, Kaplan LI. Studies in myasthenia gravis; preliminary report on therapy with mestinon bromide. J Am Med Assoc 1954;155(11):961-5. [PubMed: 13162833]

9. Schwab RS, Timberlake WH. Pyridostigmin (mestinon) in the treatment of myasthenia gravis. N Engl J Med 1954;251(7):271-2. [PubMed: 13185412]

10. Tether JE. Mestinon in myasthenia gravis; preliminary report. Dis Nerv Syst 1954;15(8):227-31. [PubMed: 13191272]

11. Westerberg MR, Magee KR. Mestinon in the treatment of myasthenia gravis. Neurology 1954;4(10):762-72. [PubMed: 13214277]

12. Hatanaka Y, Hemmi S, Morgan MB, et al. Nonresponsiveness to anticholinesterase agents in patients with MuSK-antibody-positive MG. Neurology 2005; 65(9):1508-9.

13. Pasnoor M, Wolfe GI, Nations S, et al. Clinical findings in MuSK-antibody positive myasthenia gravis: a U.S. experience. Muscle Nerve 2010;41(3):370-4. [PubMed: 19882635]

14. VanderPluym J, Vajsar J, Jacob FD, et al. Clinical characteristics of pediatric myasthenia: a surveillance study. Pediatrics 2013;132(4):e939-944. [PubMed: 24019417]

15. Kupersmith MJ, Ying G. Ocular motor dysfunction and ptosis in ocular myasthenia gravis: effects of treatment. Br J Ophthalmol 2005;89(10):1330-4. [PubMed: 16170126]

16. Mittal MK, Barohn RJ, Pasnoor M, et al. Ocular myasthenia gravis in an academic neuroophthalmology clinic: clinical features and therapeutic response. J Clin Neuromuscul Dis 2011;13(1):46-52. [PubMed: 22361625]

17. Warmolts JR, Engel WK. Benefit from alternate-day prednisone in myasthenia gravis. N Engl J Med 1972;286(1):17-20. [PubMed: 4331158]

18. Jenkins RB. Treatment of myasthenia gravis with prednisone. Lancet 1972; 1(7754):765-7. [PubMed: 4111245]

19. Pascuzzi RM, Coslett HB, Johns TR. Long-term corticosteroid treatment of myasthenia gravis: report of 116 patients. Ann Neurol 1984;15(3):291-8. [PubMed: 6721451]

20. Benatar M, McDermott MP, Sanders DB, et al. Efficacy of prednisone for the treatment of ocular myasthenia (EPITOME): a randomized, controlled trial. Muscle Nerve 2016;53(3):363-9. [PubMed: 26179124]

21. Lindberg C, Andersen O, Lefvert AK. Treatment of myasthenia gravis with methylprednisolone pulse: a double blind study. Acta Neurol Scand 1998;97(6):370-3. [PubMed: 9669469]

22. Sanders DB, Wolfe GI, Benatar M, et al. International consensus guidance for management of myasthenia gravis: executive summary. Neurology 2016; 87(4):419-25. [PubMed: 27358333]

23. Gilhus NE. Myasthenia gravis. N Engl J Med 2016;375(26):2570-81. [PubMed: 28029925]

24. Seybold ME, Drachman DB. Gradually increasing doses of prednisone in myasthenia gravis. Reducing the hazards of treatment. N Engl J Med 1974;290(2):81-4. [PubMed: 4808454]

25. A trial of mycophenolate mofetil with prednisone as initial immunotherapy in myasthenia gravis. Neurology 2008;71(6):394-9. [PubMed: 18434639]

26. Benatar M, Kaminski HJ. Evidence report: the medical treatment of ocular myasthenia (an evidence-based review): report of the Quality Standards Subcommittee of the American Academy of Neurology. Neurology 2007;68(24):2144-9. [PubMed: 17460154]

27. Kupersmith MJ, Latkany R, Homel P. Development of generalized disease at 2 years in patients with ocular myasthenia gravis. Arch Neurol 2003;60(2):243-8. [PubMed: 12580710]

28. Monsul NT, Patwa HS, Knorr AM, et al. The effect of prednisone on the progression from ocular to generalized myasthenia gravis. J Neurol Sci 2004;217(2):131-3. [PubMed: 14706214]

29. Barohn RD, Dimachkie MM. Immunomodulatory therapies in myasthenia gravis In: Mazia C, editor. Miastenia gravis y trastornos relacionados. Buenos Aires (Argentina): Editorial InterMedica; 2017 p. 273-88.

30. Mertens HG, Hertel G, Reuther P, et al. Effect of immunosuppressive drugs (azathioprine). Ann N Y Acad Sci 1981;377:691-9. [PubMed: 6951493]

31. Witte AS, Cornblath DR, Parry GJ, et al. Azathioprine in the treatment of myasthenia gravis. Ann Neurol 1984;15(6):602-5. [PubMed: 6742794] 
32. Palace J, Newsom-Davis J, Lecky B. A randomized double-blind trial of prednisolone alone or with azathioprine in myasthenia gravis. Myasthenia Gravis Study Group. Neurology 1998;50(6): 1778-83. [PubMed: 9633727]

33. Mantegazza R, Antozzi C, Peluchetti D, et al. Azathioprine as a single drug or in combination with steroids in the treatment of myasthenia gravis. J Neurol 1988; 235(8):449-53. [PubMed: 3062134]

34. Sommer N, Sigg B, Melms A, et al. Ocular myasthenia gravis: response to long-term immunosuppressive treatment. J Neurol Neurosurg Psychiatry 1997;62(2):156-62. [PubMed: 9048716]

35. Hohlfeld R, Michels M, Heininger K, et al. Azathioprine toxicity during long-term immunosuppression of generalized myasthenia gravis. Neurology 1988;38(2):258-61. [PubMed: 3340289]

36. Bernatsky S, Clarke AE, Suissa S. Hematologic malignant neoplasms after drug exposure in rheumatoid arthritis. Arch Intern Med 2008;168(4):378-81. [PubMed: 18299492]

37. Booth RA, Ansari MT, Loit E, et al. Assessment of thiopurine S-methyltransferase activity in patients prescribed thiopurines: a systematic review. Ann Intern Med 2011;154(12):814-23, w-295-818. [PubMed: 21690596]

38. Simmons WD, Rayhill SC, Sollinger HW. Preliminary risk-benefit assessment of mycophenolate mofetil in transplant rejection. Drug Saf 1997;17(2):75-92. [PubMed: 9285199]

39. Hauser RA, Malek AR, Rosen R. Successful treatment of a patient with severe refractory myasthenia gravis using mycophenolate mofetil. Neurology 1998; 51(3):912-3.

40. Chaudhry V, Cornblath DR, Griffin JW, et al. Mycophenolate mofetil: a safe and promising immunosuppressant in neuromuscular diseases. Neurology 2001; 56(1):94-6. [PubMed: 11148242]

41. Meriggioli MN, Ciafaloni E, Al-Hayk KA, et al. Mycophenolate mofetil for myasthenia gravis: an analysis of efficacy, safety, and tolerability. Neurology 2003; 61(10):1438-40. [PubMed: 14638974]

42. Sanders DB, Hart IK, Mantegazza R, et al. An international, phase III, randomized trial of mycophenolate mofetil in myasthenia gravis. Neurology 2008;71(6):400-6. [PubMed: 18434638]

43. Hehir MK, Burns TM, Alpers J, et al. Mycophenolate mofetil in AChR-antibody-positive myasthenia gravis: outcomes in 102 patients. Muscle Nerve 2010;41(5):593-8. [PubMed: 20405499]

44. Mycophenolate REMS risks of first trimester pregnancy loss and congenital malformations. Available at: https://www.mycophenolaterems.com/. Accessed January 18, 2018.

45. Vernino S, Salomao DR, Habermann TM, et al. Primary CNS lymphoma complicating treatment of myasthenia gravis with mycophenolate mofetil. Neurology 2005;65(4):639-41. [PubMed: 16116136]

46. Dubal DB, Mueller S, Ruben BS, et al. T-cell lymphoproliferative disorder following mycophenolate treatment for myasthenia gravis. Muscle Nerve 2009; 39(6):849-50. [PubMed: 19358236]

47. Tindall RS, Rollins JA, Phillips JT, et al. Preliminary results of a double-blind, randomized, placebo-controlled trial of cyclosporine in myasthenia gravis. N Engl J Med 1987;316(12):719-24. [PubMed: 3547126]

48. Tindall RS, Phillips JT, Rollins JA, et al. A clinical therapeutic trial of cyclosporine in myasthenia gravis. Ann N Y Acad Sci 1993;681:539-51. [PubMed: 8357194]

49. Ciafaloni E, Nikhar NK, Massey JM, et al. Retrospective analysis of the use of cyclosporine in myasthenia gravis. Neurology 2000;55(3):448-50. [PubMed: 10932288]

50. Nagane Y, Utsugisawa K, Obara D, et al. Efficacy of low-dose FK506 in the treatment of Myasthenia gravis-a randomized pilot study. Eur Neurol 2005;53(3):146-50. [PubMed: 15900097]

51. Liu C, Gui M, Cao Y, et al. Tacrolimus improves symptoms of children with myasthenia gravis refractory to prednisone. Pediatr Neurol 2017;77:42-7. [PubMed: 29074055]

52. Brown PM, Pratt AG, Isaacs JD. Mechanism of action of methotrexate in rheumatoid arthritis, and the search for biomarkers. Nat Rev Rheumatol 2016; 12(12):731-42. [PubMed: 27784891] 
53. Heckmann JM, Rawoot A, Bateman K, et al. A single-blinded trial of methotrexate versus azathioprine as steroid-sparing agents in generalized myasthenia gravis. BMC Neurol 2011;11:97. [PubMed: 21819556]

54. Pasnoor M, He J, Herbelin L, et al. A randomized controlled trial of methotrexate for patients with generalized myasthenia gravis. Neurology 2016;87(1):57-64. [PubMed: 27306628]

55. Perez MC, Buot WL, Mercado-Danguilan C, et al. Stable remissions in myasthenia gravis. Neurology 1981;31(1):32-7. [PubMed: 7192824]

56. De Feo LG, Schottlender J, Martelli NA, et al. Use of intravenous pulsed cyclophosphamide in severe, generalized myasthenia gravis. Muscle Nerve 2002; 26(1):31-6. [PubMed: 12115946]

57. Drachman DB, Jones RJ, Brodsky RA. Treatment of refractory myasthenia: "rebooting" with highdose cyclophosphamide. Ann Neurol 2003;53(1):29-34. [PubMed: 12509845]

58. Tandan R, Hehir MK 2nd, Waheed W, et al. Rituximab treatment of myasthenia gravis: a systematic review. Muscle Nerve 2017;56(2):185-96. [PubMed: 28164324]

59. Zaja F, Russo D, Fuga G, et al. Rituximab for myasthenia gravis developing after bone marrow transplant. Neurology 2000;55(7):1062-3.

60. Vander Heiden JA, Stathopoulos P, Zhou JQ, et al. Dysregulation of B cell repertoire formation in myasthenia gravis patients revealed through deep sequencing. J Immunol 2017;198(4):1460-73. [PubMed: 28087666]

61. Collongues N, Casez O, Lacour A, et al. Rituximab in refractory and nonrefractory myasthenia: a retrospective multicenter study. Muscle Nerve 2012; 46(5):687-91. [PubMed: 22941747]

62. Hain B, Jordan K, Deschauer M, et al. Successful treatment of MuSK antibody-positive myasthenia gravis with rituximab. Muscle Nerve 2006;33(4):575-80. [PubMed: 16323216]

63. Diaz-Manera J, Martinez-Hernandez E, Querol L, et al. Long-lasting treatment effect of rituximab in MuSK myasthenia. Neurology 2012;78(3):189-93. [PubMed: 22218276]

64. Silvestri NJ, Wolfe GI. Myasthenia gravis. Semin Neurol 2012;32(3):215-26. [PubMed: 23117946]

65. Kanth KM, Solorzano GE, Goldman MD. PML in a patient with myasthenia gravis treated with multiple immunosuppressing agents. Neurol Clin Pract 2016;6(2):e17-9. [PubMed: 27104071]

66. Vermeer NS, Straus SM, Mantel-Teeuwisse AK, et al. Drug-induced progressive multifocal leukoencephalopathy: lessons learned from contrasting natalizumab and rituximab. Clin Pharmacol Ther 2015;98(5):542-50. [PubMed: 26347128]

67. Nowak R, Coffey C, Goldstein J, et al. A phase 2 trial of rituximab in myasthenia gravis: study update. 14th International Congress on Neuromuscular Diseases (ICNMD XIV). Toronto (Canada), 7 7, 2016.

68. Pinching AJ, Peters DK. Remission of myasthenia gravis following plasma-exchange. Lancet 1976;2(8000):1373-6. [PubMed: 63848]

69. Dau PC, Lindstrom JM, Cassel CK, et al. Plasmapheresis and immunosuppressive drug therapy in myasthenia gravis. N Engl J Med 1977;297(21):1134-40. [PubMed: 917042]

70. Plasmapheresis and acute Guillain-Barre syndrome. The Guillain-Barre syndrome Study Group. Neurology 1985;35(8):1096-104. [PubMed: 4022342]

71. Patwa HS, Chaudhry V, Katzberg H, et al. Evidence-based guideline: intravenous immunoglobulin in the treatment of neuromuscular disorders: report of the Therapeutics and Technology Assessment Subcommittee of the American Academy of Neurology. Neurology 2012;78(13): 1009-15. [PubMed: 22454268]

72. Gajdos P, Chevret S, Toyka K. Plasma exchange for myasthenia gravis. Cochrane Database Syst Rev 2002;(4):CD002275. [PubMed: 12519572]

73. Barth D, Nabavi Nouri M, Ng E, et al. Comparison of IVIg and PLEX in patients with myasthenia gravis. Neurology 2011;76(23):2017-23. [PubMed: 21562253]

74. Zinman L, Ng E, Bril V. IV immunoglobulin in patients with myasthenia gravis: a randomized controlled trial. Neurology 2007;68(11):837-41. [PubMed: 17353471]

75. Ebadi H, Barth D, Bril V. Safety of plasma exchange therapy in patients with myasthenia gravis. Muscle Nerve 2013;47(4):510-4. [PubMed: 23322564] 
76. Yeh JH, Wang SH, Chien PJ, et al. Changes in serum cytokine levels during plasmapheresis in patients with myasthenia gravis. Eur J Neurol 2009;16(12):1318-22. [PubMed: 19614971]

77. Mandawat A, Mandawat A, Kaminski HJ, et al. Outcome of plasmapheresis in myasthenia gravis: delayed therapy is not favorable. Muscle Nerve 2011; 43(4):578-84. [PubMed: 21404289]

78. Liew WK, Powell CA, Sloan SR, et al. Comparison of plasmapheresis and intravenous immunoglobulin as maintenance therapies for juvenile myasthenia gravis. JAMA Neurol 2014;71(5):575-80. [PubMed: 24590389]

79. Shemin D, Briggs D, Greenan M. Complications of therapeutic plasma exchange: a prospective study of 1,727 procedures. J Clin Apher 2007;22(5):270-6. [PubMed: 17722046]

80. Ahmed F, Vamanan K, Dimachkie M, et al. Arteriovenous fistula venous access for long-term outpatient plasma exchange for neuromuscular disorders. American Academy of Neurology Annual Meeting Seattle (WA), April 25 - May 02, 2009.

81. Gajdos P, Outin H, Elkharrat D, et al. High-dose intravenous gammaglobulin for myasthenia gravis. Lancet 1984;1(8373):406-7.

82. Arsura EL, Bick A, Brunner NG, et al. High-dose intravenous immunoglobulin in the management of myasthenia gravis. Arch Intern Med 1986;146(7):1365-8. [PubMed: 3718134]

83. Achiron A, Barak Y, Miron S, et al. Immunoglobulin treatment in refractory myasthenia gravis. Muscle Nerve 2000;23(4):551-5. [PubMed: 10716766]

84. Wolfe GI, Barohn RJ, Foster BM, et al. Randomized, controlled trial of intravenous immunoglobulin in myasthenia gravis. Muscle Nerve 2002;26(4):549-52. [PubMed: 12362423]

85. Gajdos P, Chevret S, Clair B, et al. Clinical trial of plasma exchange and high-dose intravenous immunoglobulin in myasthenia gravis. Myasthenia Gravis Clinical Study Group. Ann Neurol 1997;41(6):789-96. [PubMed: 9189040]

86. Gajdos P, Tranchant C, Clair B, et al. Treatment of myasthenia gravis exacerbation with intravenous immunoglobulin: a randomized double-blind clinical trial. Arch Neurol 2005;62(11): 1689-93. [PubMed: 16286541]

87. Dalakas MC. Intravenous immunoglobulin in autoimmune neuromuscular diseases. JAMA 2004;291(19):2367-75. [PubMed: 15150209]

88. Brannagan TH 3rd, Nagle KJ, Lange DJ, et al. Complications of intravenous immune globulin treatment in neurologic disease. Neurology 1996;47(3):674-7. [PubMed: 8797463]

89. Barohn RJ, Brey RL. Soluble terminal complement components in human myasthenia gravis. Clin Neurol Neurosurg 1993;95(4):285-90. [PubMed: 8299285]

90. Engel AG, Arahata K. The membrane attack complex of complement at the end-plate in myasthenia gravis. Ann N Y Acad Sci 1987;505:326-32. [PubMed: 3318619]

91. Engel AG, Lambert EH, Howard FM. Immune complexes (IgG and C3) at the motor end-plate in myasthenia gravis: ultrastructural and light microscopic localization and electrophysiologic correlations. Mayo Clin Proc 1977;52(5):267-80. [PubMed: 870771]

92. Howard JF Jr, Barohn RJ, Cutter GR, et al. A randomized, double-blind, placebo-controlled phase II study of eculizumab in patients with refractory generalized myasthenia gravis. Muscle Nerve 2013;48(1):76-84. [PubMed: 23512355]

93. Gronseth GS, Barohn RJ. Practice parameter: thymectomy for autoimmune myasthenia gravis (an evidence-based review): report of the Quality Standards Subcommittee of the American Academy of Neurology. Neurology 2000;55(1):7-15. [PubMed: 10891896]

94. Cea G, Benatar M, Verdugo RJ, et al. Thymectomy for non-thymomatous myasthenia gravis. Cochrane Database Syst Rev 2013;(10):CD008111. [PubMed: 24122674]

95. Kissel JT, Franklin GM. Treatment of myasthenia gravis: a call to arms. Neurology 2000;55(1):34. [PubMed: 10891894]

96. Wolfe GI, Kaminski HJ, Aban IB, et al. Randomized trial of thymectomy in myasthenia gravis. N Engl J Med 2016;375(6):511-22. [PubMed: 27509100]

97. Skeie GO, Apostolski S, Evoli A, et al. Guidelines for treatment of autoimmune neuromuscular transmission disorders. Eur J Neurol 2010;17(7):893-902. [PubMed: 20402760] 
98. Goldstein SD, Culbertson NT, Garrett D, et al. Thymectomy for myasthenia gravis in children: a comparison of open and thoracoscopic approaches. J Pediatr Surg 2015;50(1):92-7. [PubMed: 25598101]

99. Wagner AJ, Cortes RA, Strober J, et al. Long-term follow-up after thymectomy for myasthenia gravis: thoracoscopic vs open. J Pediatr Surg 2006;41(1):50-4 [discussion: 50-4]. [PubMed: 16410107]

100. Jaretzki A 3rd, Barohn RJ, Ernstoff RM, et al. Myasthenia gravis: recommendations for clinical research standards. Task Force of the Medical Scientific Advisory Board of the Myasthenia Gravis Foundation of America. Neurology 2000; 55(1):16-23. [PubMed: 10891897]

101. Ronager J, Ravnborg M, Hermansen I, et al. Immunoglobulin treatment versus plasma exchange in patients with chronic moderate to severe myasthenia gravis. Artif Organs 2001;25(12):967-73. [PubMed: 11843764]

102. Sanders DB, Rosenfeld J, Dimachkie MM, et al. A double-blinded, randomized, placebocontrolled trial to evaluate efficacy, safety, and tolerability of single doses of tirasemtiv in patients with acetylcholine receptor-binding antibody-positive myasthenia gravis.

Neurotherapeutics 2015;12(2):455-60. [PubMed: 25742919] 


\section{KEY POINTS}

- With advances in myasthenia gravis treatment, most patients have very good outcomes. The bedrock of MG treatment is immunotherapy, and symptomatic treatment with acetylcholinesterase inhibition.

- A recent international, rater-blinded, randomized trial provided strong evidence of improved clinical outcomes in acetylcholine receptor antibody positive nonthymomatous myasthenia gravis treated with thymectomy.

- In ocular disease, a randomized controlled trial found corticosteroids to be beneficial. Another recent trial failed to show a steroid-sparing effect in patients treated with methotrexate.

- A complement inhibitor, eculizumab was recently approved for the treatment of generalized myasthenia gravis. There are emerging therapies, including targeted monoclonal antibody agents that are currently under investigation.

- Patient recruitment continues to be a challenge in myasthenia gravis clinical trials. 


\section{Box 1}

\section{Myasthenia gravis treatment: controlled randomized trials}

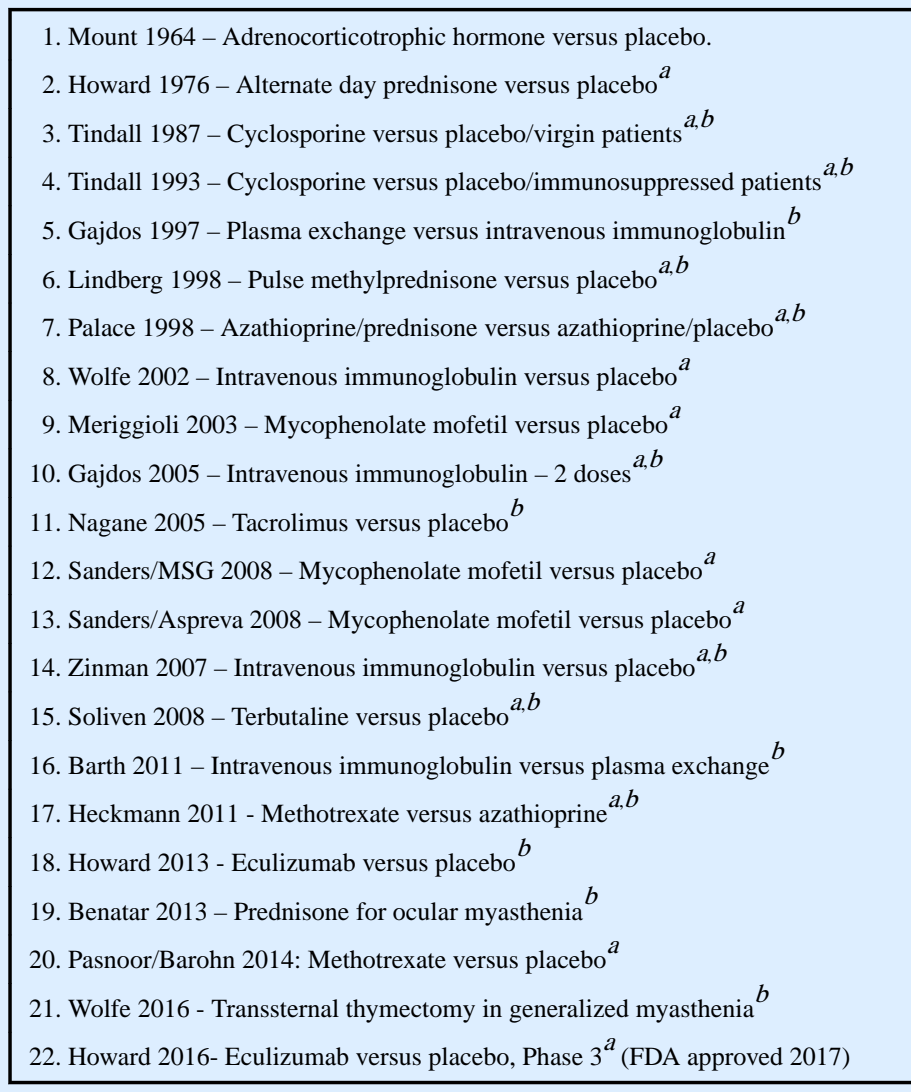

${ }^{a}$ Blinded.

${ }^{b}$ Positive trials. 


\section{Box 2}

Treatments for myasthenia gravis and decade introduced

1930s: physostigmine, neostigmine

1940s: thymectomy

1950s: mechanical ventilation, edrophonium chloride, pyridostigmine

1960s: corticosteroids and plasma exchange

1970s: azathioprine

1980s: cyclosporine, cyclophosphamide

1990s: intravenous immunoglobulin

2000s: mycophenolate mofetil, tacrolimus

2010s: rituximab, eculizumab 


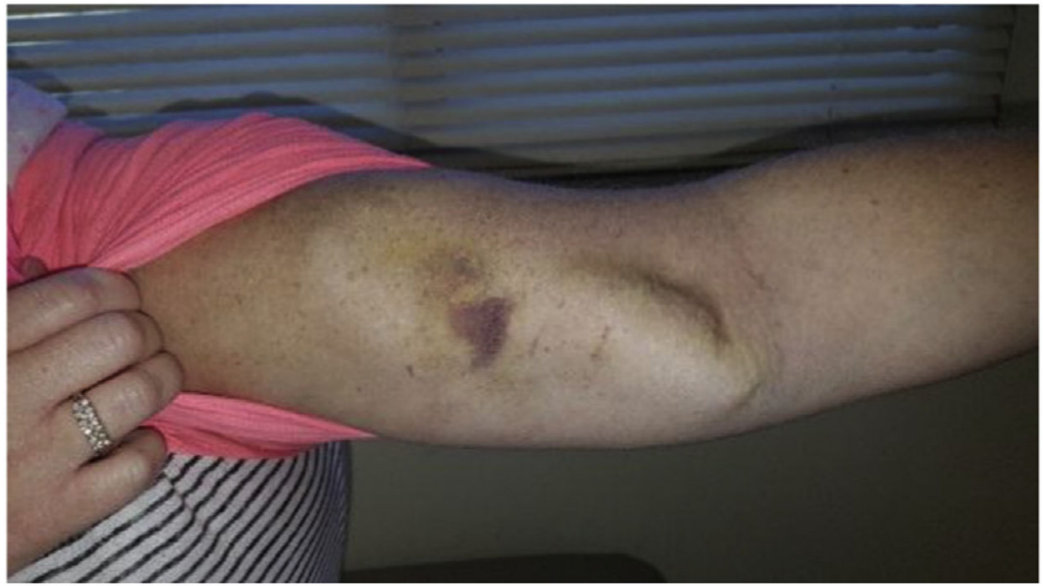

Fig. 1.

Arteriovenous fistula for plasma exchange in myasthenia gravis. 


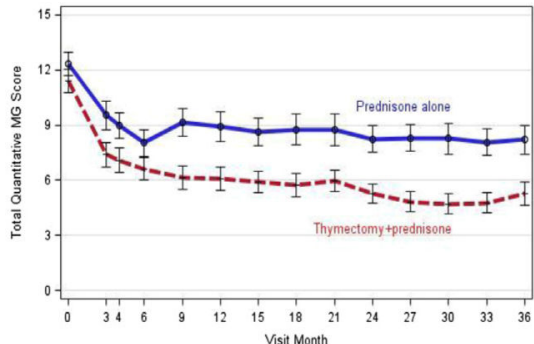

QMG Score (Mean \pm SE) by Treatment Group

- QMG difference: 2.85 pts (99.5\% Cl 0.47-5.22; $P<.001$ )

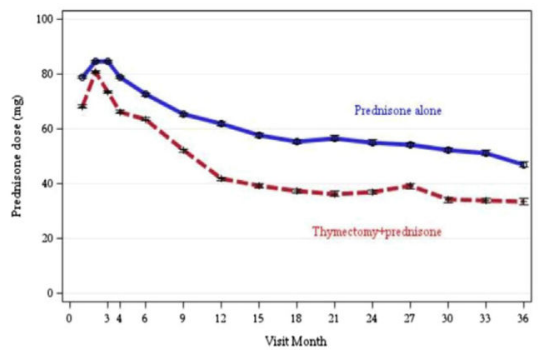

Time-Weighted Average AD

Prednisone Dose (Mean \pm SE) by

Treatment Group

- Prednisone dose difference:32 mg vs 54 mg (95\% Cl 12-32 mg; $P<.001)$

Fig. 2.

Wolfe et al thymectomy in MG. (Data from New England Journal of Medicine 2016;375(6): 511-522.) 

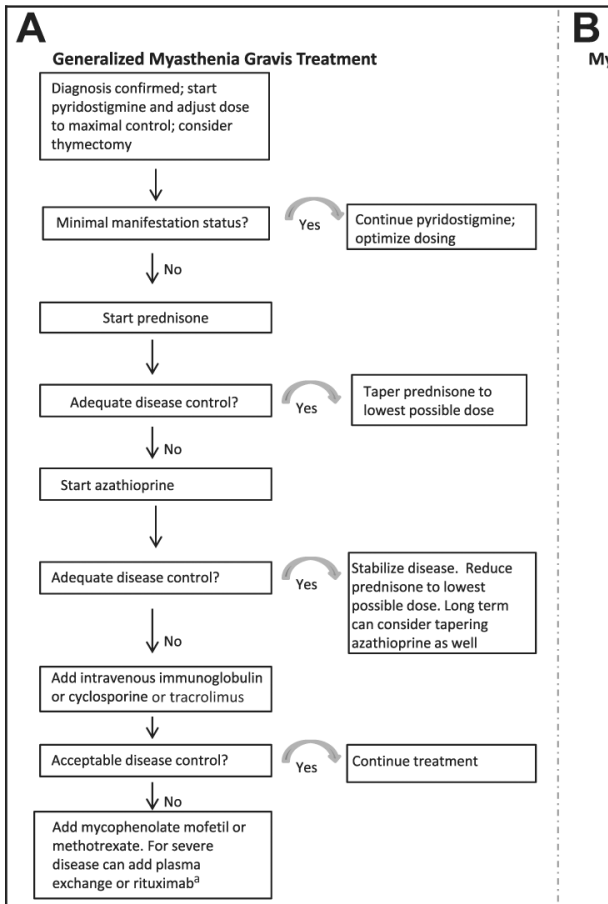

B

Myasthenic Crisis and Severe Exacerbation Treatment

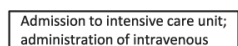

administration of intravenous

exchange, increase steroid

exchange, increase steroid
dose and stop pyridostigmine

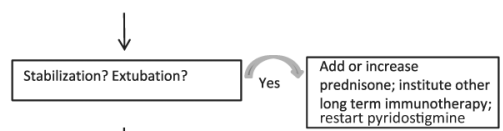

Administration of plasma Administration of plasma
exchange or intravenous immunoglobulin

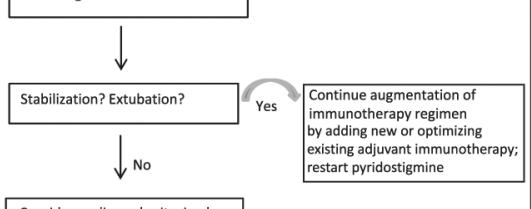

Consider eculizumab, rituximab,

dose steroids; continue trying othe

immunosuppressive agents

Fig. 3.

Suggested algorithms for the treatment of generalized myasthenia gravis and myasthenic crisis. (A) Generalized myasthenia gravis treatment. $(B)$ Myasthenic crisis and severe exacerbation treatment. ${ }^{\text {a }}$ If not better, consider eculizumab. 


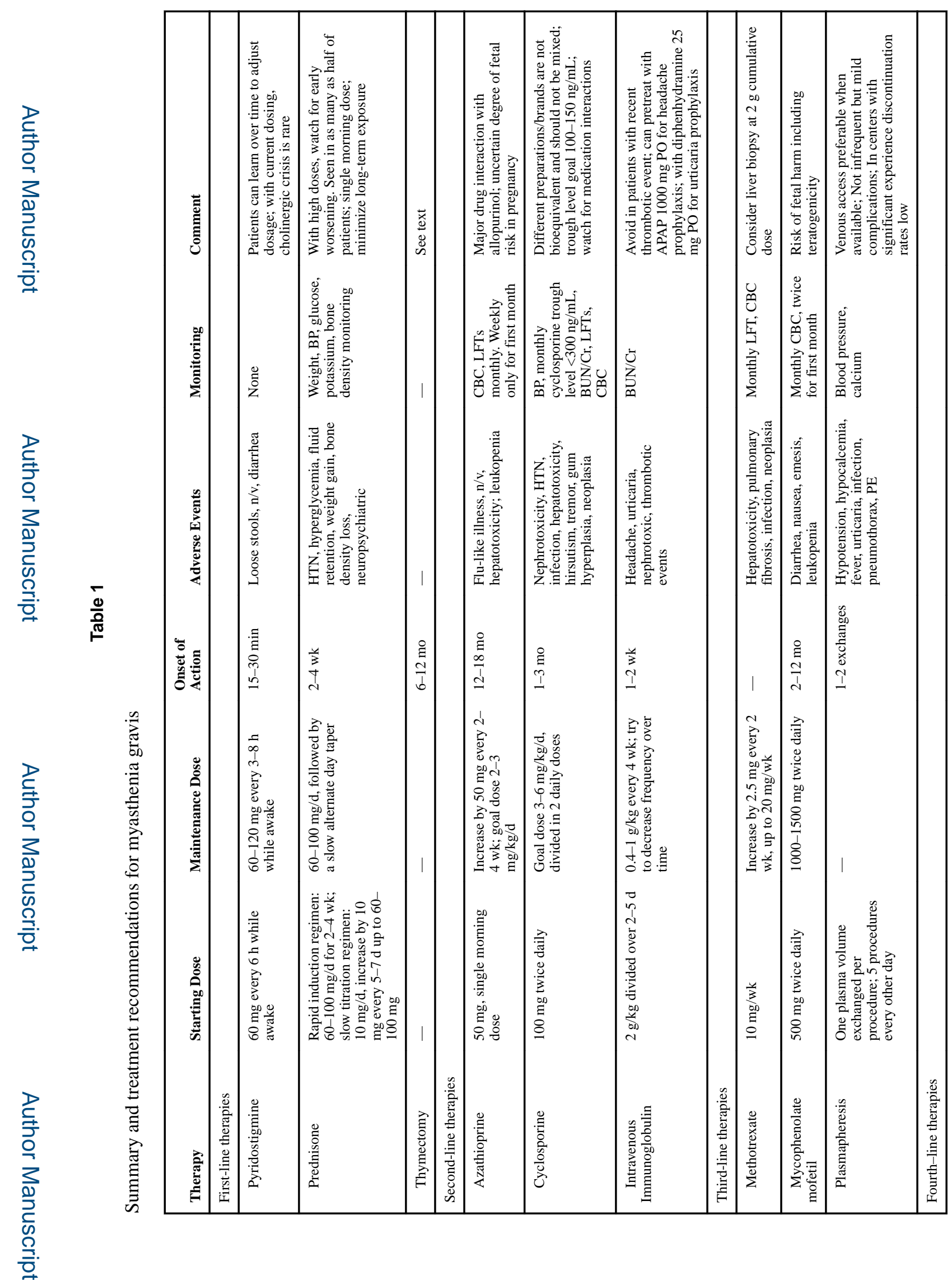

Neurol Clin. Author manuscript; available in PMC 2019 August 12. 


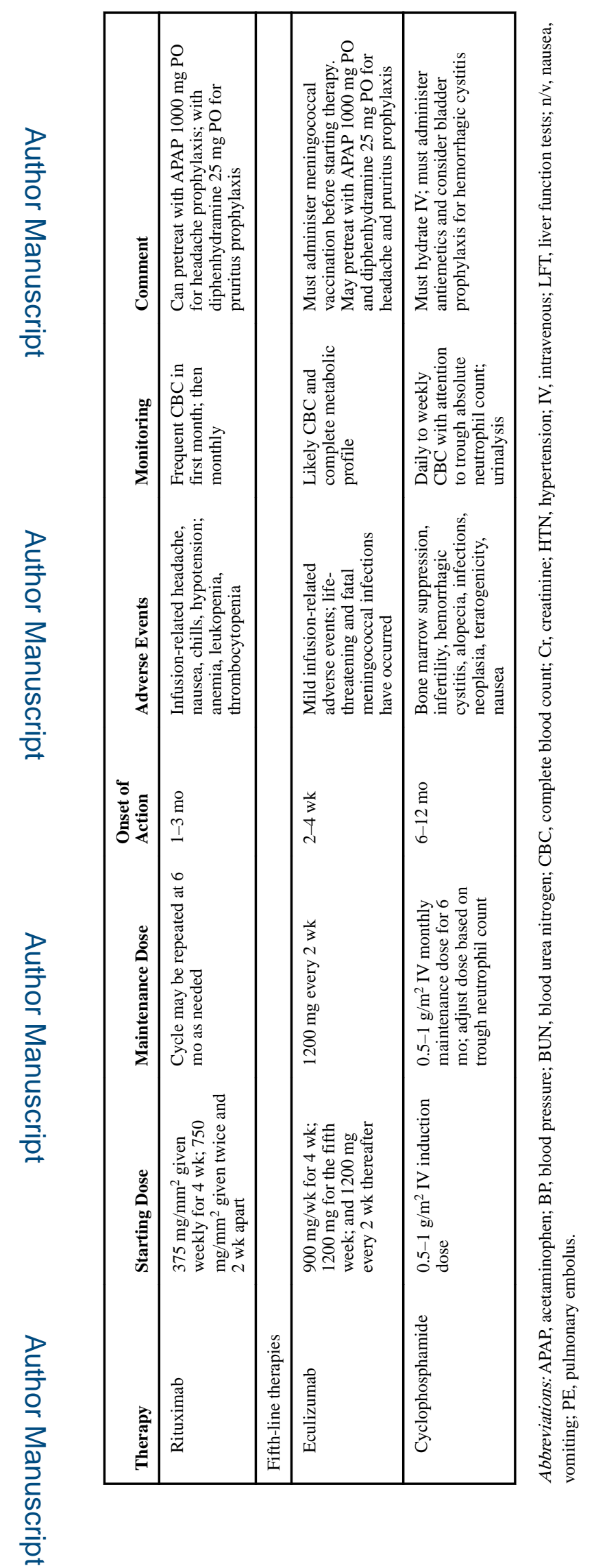

Neurol Clin. Author manuscript; available in PMC 2019 August 12. 


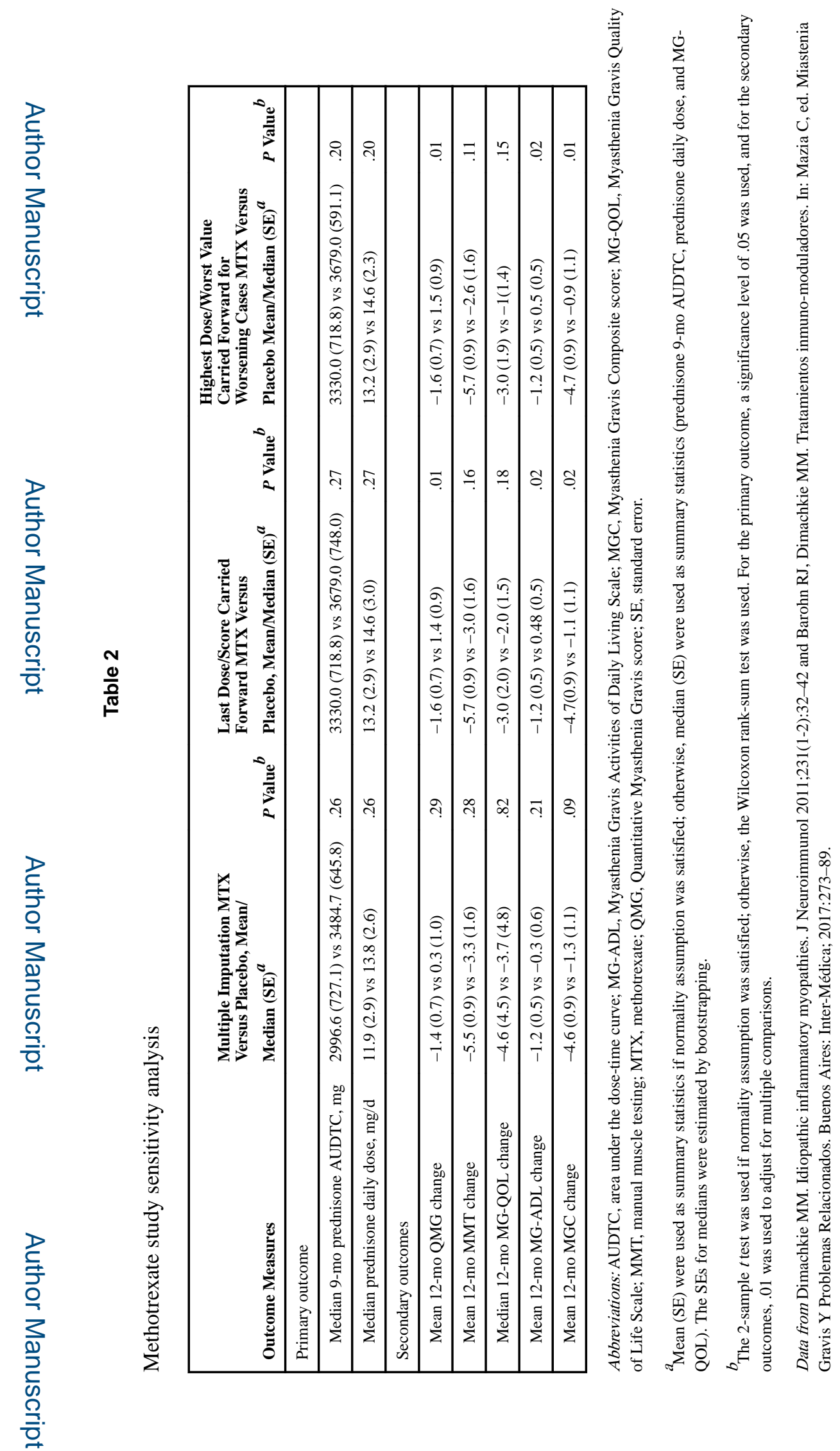

Neurol Clin. Author manuscript; available in PMC 2019 August 12. 
Table 3

Thymectomy Procedures

\begin{tabular}{|ll|}
\hline Type & Year \\
\hline Sternal splitting & Early 1900s \\
\hline Maximally invasive & $1980 \mathrm{~s}$ \\
\hline Transcervical & 1988 \\
\hline Video-assisted thoracoscopic surgery & Late 1990s \\
\hline Robotics (DaVinci) & Early 2000s \\
\hline
\end{tabular}




\section{Table 4}

Treatment recommendations for myasthenia gravis

\begin{tabular}{|ll|}
\hline Prior to 2007 & $\mathbf{2 0 1 8}$ \\
\hline 1st Line & \\
Tensilon ${ }^{\circledR}$ & Enlon ${ }^{\circledR}$ \\
Pyridostigmine & Pyridostigmine \\
Prednisone & Prednisone \\
Thymectomy? & Thymectomy! YES \\
2nd Line & \\
Azathioprine & Azathioprine \\
Mycophenolate mofetil & Cyclosporine/tacrolimus \\
Cyclosporine & IVIG \\
3rd Line & \\
IVIG & Plasma exchange \\
Plasma exchange & Mycophenolate mofetil Methotrexate \\
4th Line & \\
None & Eculizumab (Soliris $\left.{ }^{\circledR}\right)$ Rituximab \\
5th Line & \\
None & Cyclophosphamide \\
\hline
\end{tabular}


Table 5

Advantages and disadvantages of IVIG versus PLEX in MG

\begin{tabular}{|llll|}
\hline Pro-PLEX & Con-PLEX & Pro-IVIG & Con-IVIG \\
\hline Probably effective & No RCT & Positive RCT & $\begin{array}{l}\text { Insurance coverage limitations; not FDA } \\
\text { approved for MG }\end{array}$ \\
\hline Longer track record & Morbidity; need for central access & Ease of use & Shorter track record \\
\hline May work faster & $\begin{array}{l}\text { Sophisticated equipment; need for } \\
\text { trained staff }\end{array}$ & Generally well-tolerated & $\begin{array}{l}\text { Rare side effects: anaphylaxis, kidney injury, } \\
\text { thrombosis }\end{array}$ \\
\hline $\begin{array}{l}\text { Abbreviations: FDA, US Food and Drug Administration; IVIG, intravenous immunoglobulin; MG, myasthenia gravis; PLEX, plasma exchange; } \\
\text { RCT, randomized, controlled trial. }\end{array}$
\end{tabular}

